\title{
OntoPilot: New Software to Simplify and Accelerate Ontology Development and Deployment
}

\author{
Brian J Stucky $\ddagger$, Annie Luc $§$ \\ ‡ Florida Museum of Natural History, University of Florida, Gainesville, Florida, United States of America \\ § University of Florida, Gainesville, Florida, United States of America
}

Corresponding author: Brian J Stucky (stuckyb@flmnh.ufl.edu)

Received: 11 Aug 2017 | Published: 11 Aug 2017

Citation: Stucky B, Luc A (2017) OntoPilot: New Software to Simplify and Accelerate Ontology Development and Deployment. Proceedings of TDWG 1: e20192. https://doi.org/10.3897/tdwgproceedings.1.20192

\begin{abstract}
Ontologies (formal descriptions of the concepts and relationships that can exist in knowledge domains) provide the conceptual foundations for modern knowledge representation systems and knowledge bases, and they are a core component of automated reasoning pipelines that infer new facts from existing data. Although a variety of software tools are available for authoring and manipulating ontologies, ontology development remains a technically demanding, time consuming, and often tedious task. In this talk, I will introduce OntoPilot, new software that endeavors to at least partially replace the current patchwork of disparate ontology development tools with a single, unified development framework that is easy to learn and requires minimal knowledge of the technical details of ontology description languages. OntoPilot uses familiar tabular document formats (e.g., spreadsheets) as ontology "source code", which means that domain experts without detailed knowledge of ontology-related technologies can still directly participate in the development process. OntoPilot supports a complete ontology development workflow, including reusing terms from external ontologies, defining new terms, managing releases, and generating end-user documentation. Furthermore, OntoPilot makes it easy to build inference pipelines that use ontologies to generate novel insights from existing data. OntoPilot is open-source software, supports all major operating systems, and is freely available at https://github.com/stuckyb/ontopilot/.
\end{abstract}




\section{Keywords}

ontology development, knowledge representation, knowledge engineering

\section{Presenting author}

Brian J. Stucky 